\title{
Davies Critical Point and Tunneling
}

\author{
HoSeong La* \\ Department of Physics and Astronomy, \\ The University of Tennessee, \\ Knoxville, TN 37996, USA \\ hsla.avt@gmail.com
}

\begin{abstract}
From the point of view of tunneling, the physical meaning of the Davies critical point of a second order phase transition in the black hole thermodynamics is clarified. At the critical point, the nonthermal contribution vanishes so that the black hole radiation is entirely thermal. It separates two phases: one with radiation enhanced by the nonthermal contribution, the other suppressed by the nonthermal contribution. We show this in both charged and rotating black holes. The phase transition is also analyzed in the cases in which emissions of charges and angular momenta are incorporated.
\end{abstract}

\footnotetext{
* Present address: Department of Physics and Astronomy, Vanderbilt University, Nashville, TN 37235, USA
} 


\section{INTRODUCTION}

Upon the discovery of the thermodynamic interpretation of black holes[1], the first critical phenomenon identified was the second order phase transition discovered by Davies more than thirty years ago[2]. The phase structure was analyzed by Hut[3], and it has been generalized for varying charges of isolated charged black holes[4]. The issue of stability in the Kerr black hole case has been addressed[5]. Yet, all these are still in the thermal radiation context based on the Hawking process. Here we present a new proposal to clarify the physical meaning of this critical phenomenon in the context of the nonthermal radiation of Parikh-Wilczek[6][7]. The existence of nonthermal radiation from black holes have been anticipated[8][9] beyond Hawking's thermal radiation[10].

Black holes are unusual thermodynamic systems because we cannot see any structural changes directly as far as critical phenomena are concerned. The entropy changes smoothly so that checking the behavior of the horizon cannot tell us either. So the only way to tell what is going on in the black hole phases is if there is any difference in emissions of information from different phases. So the tunneling argument of Parikh-Wilczek is a good candidate to investigate if there are such emissions telling about the black holes phases. In this paper, we find that indeed this is the case, and that, using this information, we can explain the physical meaning of the Davies critical phenomenon.

In short, we find that there is a competition between thermal part of radiation and nonthermal part. At the critical point, the nonthermal part vanishes, leaving only thermal radiation that peaks. The critical point separates two phases: In one phase, the nonthermal contribution enhances the total radiation, while in the other phase the nonthermal contribution actually suppresses the total radiation. Once we extend to the cases of emissions of charges or angular momenta, we can observe over all enhancement or suppression, but the peak of emission remains at (or near) the critical point and separation of two phases persists. We could check the characteristics of each phase of black holes with respect to the radiation and emissions of charges and angular momenta by introducing effective free energies.

This paper is organized as following. In section 2, we review and identify what the Davies critical point is. In section 3, we explain this critical phenomenon in the RN (ReissnerNordtröm) black hole case, using the tunneling argument. Then, in section 4, it is extended to incorporate the emissions of charges and angular momenta in the KN (Kerr-Newman) 
case. Finally, in section 5, some further comments are given.

\section{DAVIES CRITICAL POINT}

The Davies critical point is identified by the singular behavior of specific heat at some nontrivial value of charge-to-mass ratio, $Q / M$, or, angular-momentum-to-mass ratio, $J / M^{2}$, away from the extremal limit[2]. A second order phase transition occurs at this critical point and the phenomenon is generic for any charged or rotating black holes. The nature of this critical phenomenon is not entirely clear except it has been known that the specific heat changes the sign abruptly.

To illustrate the Davies critical point, we shall start with the RN black hole. Fig.1 shows the relationship between the Bekenstein-Hawking entropy and the Hawking temperature for some value of charge $Q$, and, in this case, the Davies critical point is the turning point

marked by II. At the critical point, $|Q| / M_{c}=\sqrt{3} / 2, T_{c}=1 /\left(9 \pi M_{c}\right)$ and $S_{c}=(9 / 4) \pi M_{c}^{2}$ for a given charge $Q$. As $Q$ varies, the critical points trace along $S_{c} \propto 1 / T_{c}^{2}$. As one can see in the plot, specific heat, which is related to the slope of the curve, $\partial S / \partial T$, is singular at II, indicating the occurrence of some kind of a second order phase transition. The specific heat is negative in region I and positive in region III. The critical exponent for the specific heat is $1 / 2$ such that, as $T_{H}$ approaches $T_{c}$,

$$
\left|c_{Q}\right| \sim\left(T_{c}-T_{H}\right)^{-1 / 2}
$$

The Helmholtz free energy of RN black hole (fig.2) shows a cusp formed at the Davies critical point. Note that, unlike normal thermodynamic systems, the part with negative specific heat of Helmholtz free energy is convex. The Gibbs free energy also displays a turning point at the Davies critical point. In the extremal limit, Gibbs free energy vanishes for RN black hole (fig.3), but in the KN case Gibbs free energy no longer vanishes due to the rotational degrees of freedom (fig.4).

Compared with the Schwarzschild black hole case, in which the specific heat is always negative and temperature does not have a turning point, in the RN or KN case there must be something happening to generate such a turning point that the behavior of the temperature changes, at the same time, changing the specific heat from negative to positive. 


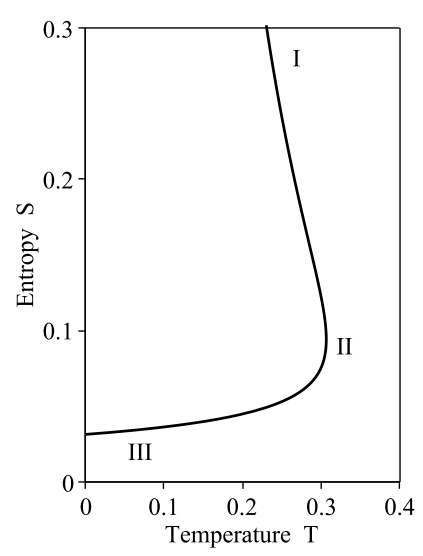

FIG. 1. S vs. T plot of RN black hole for fixed $Q=0.1$ : The turning point II is Davies critical point

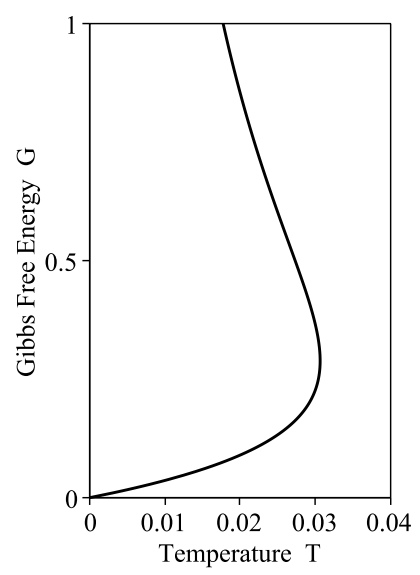

FIG. 3. RN case: At $T=0, G=0$.

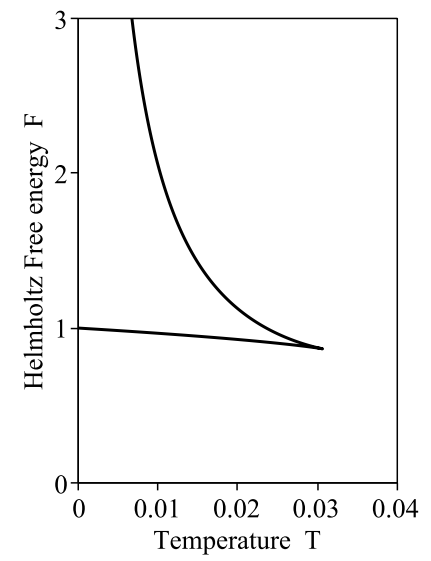

FIG. 2. Helmholtz free energy of RN black hole for fixed $Q=1$ : The end point of the cusp is Davies critical point

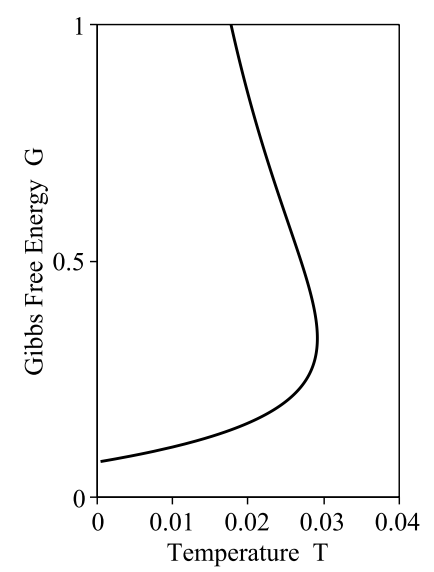

FIG. 4. KN case: At $T=0, G>0$.

\section{DAVIES CRITICAL POINT FROM TUNNELING IN REISSNER-NORDSTRÖM BLACK HOLE}

The appearance of such a charge-to-mass ratio as a critical point away from the extremal limit is quite intriguing and it certainly calls for deeper understanding of its physical implications. In this paper, We argue that what happens is due to the nonthermal black hole radiation. This can be seen by using Parikh-Wilczek's tunneling argument[6] of black hole radiation as follows. 
In tunneling, the emission rate is given by

$$
\begin{aligned}
\Gamma & \sim \exp (-2 \operatorname{Im} I) \\
& =\exp \left(-2 \operatorname{Im} \int p_{r} d r\right) \\
& =\exp \left(-2 \operatorname{Im} \int_{M}^{M-\omega} \frac{1}{2} \beta_{\mathrm{M}}(H) d H\right),
\end{aligned}
$$

where

$$
\beta_{\mathrm{M}}(H) \equiv 2 \int_{r_{\text {in }}}^{r_{\text {out }}} \frac{d r}{\dot{r}}
$$

is related to the inverse Hawking temperature as we shall see later. After integrating, which is done by Parikh-Wilczek, we get

$$
\Gamma \sim \mathrm{e}^{-\beta_{\mathrm{eff}}(\omega) \omega}
$$

where

$$
\begin{aligned}
\frac{1}{2 \pi} \beta_{\mathrm{eff}}(\omega) \equiv-\frac{1}{\omega}( & (M-\omega)^{2}+(M-\omega) \sqrt{(M-\omega)^{2}-Q^{2}} \\
& \left.-M^{2}-M \sqrt{M^{2}-Q^{2}}\right)
\end{aligned}
$$

$T_{\text {eff }}=1 / \beta_{\text {eff }}$ is called the effective temperature, which is interpreted as the indication how temperature changes after the emission of radiation. Note that this effective temperature is not the temperature after the emission of radiation, but it is a quantity still defined at the temperature before the emission. Assuming $1 \gg \omega / M$ and $1 \gg\left(2 \omega M-\omega^{2}\right) /\left(M^{2}-Q^{2}\right)$, we can expand $\beta_{\text {eff }}(\omega)$ as

$$
\frac{1}{2 \pi} \beta_{\mathrm{eff}}(\omega)=\frac{1}{2 \pi T_{H}}-\omega f(M, Q)+\text { h.o. }
$$

where

$$
T_{H}=1 / \beta_{H}=\frac{1}{2 \pi} \frac{\sqrt{M^{2}-Q^{2}}}{\left(M+\sqrt{M^{2}-Q^{2}}\right)^{2}}
$$

is the Hawking temperature and

$$
f(M, Q) \equiv 1+\frac{3}{2} \frac{M}{\left(M^{2}-Q^{2}\right)^{1 / 2}}-\frac{1}{2} \frac{M^{3}}{\left(M^{2}-Q^{2}\right)^{3 / 2}} .
$$

The key observation here is that the sign of $f(M, Q)$ depends on $M$ and $Q$. In fact, $f(M, Q)=0$ for $|Q| / M_{c}=\sqrt{3} / 2$, which is nothing but the Davies critical value! Note that $f(M, Q) \gtrless 0$ for $M \gtrless M_{c}$. 
If we can relate $f(M, Q)$ to the specific heat, we should be able to understand this better. Indeed, in terms of specific heat

$$
\begin{aligned}
c_{Q} & \equiv \frac{1}{M}\left(\frac{\partial M}{\partial T}\right)_{Q} \\
& =2 \pi \frac{\left(M+\sqrt{M^{2}-Q^{2}}\right)^{2} \sqrt{M^{2}-Q^{2}}}{M^{2}-2 M \sqrt{M^{2}-Q^{2}}} \\
& =-\frac{\beta_{H}^{2}}{4 \pi M} \frac{1}{f(M, Q)},
\end{aligned}
$$

we can reorganize eq.(6) as

$$
\beta_{\mathrm{eff}}(\omega)=\beta_{H}+\frac{1}{2} \frac{\omega}{M} \frac{\beta_{H}^{2}}{c_{Q}}+\mathcal{O}\left(\omega^{2}\right) .
$$

This clearly shows how Davies critical point appears in the tunneling context. As we shall show later, this is generic for any black hole with the corresponding specific heat. Now one can see that, for $c_{Q}<0, \beta_{\text {eff }}<\beta_{H}$ so that (effective) temperature rises, and, at the Davies critical point, $\beta_{\text {eff }}=\beta_{H}$, but as soon as black hole crosses over the Davies point into the region of $c_{Q}>0$, the temperature turns lower.

This also means the following: Ahead of the Davies point (from the right side in fig.5), $c_{Q}<0$ and the (emission rate of) radiation is enhanced by nonthermal radiation on top of the thermal radiation. At the Davies point, the specific heat is singular and the radiation is purely thermal. But as the black hole crosses the Davies point, $c_{Q}>0$ and the radiation is reduced by the nonthermal part, lowering the temperature. Even though the assumption for this tunneling, $(M-\omega)>Q$, excludes the extremal case (the solid line in fig.5 starts off away from the bottom), but the final formula still seems to make sense in the extremal limit, where the emission rate does become zero as we expect. So, we can safely extrapolate this to the extremal limit with caution. Note that the thermal radiation peaks at the Davies point and the total emission rate peaks at $M=M_{c}+\omega$. Since $\omega$ is small enough compared to $M$, it is safe to say that the total radiation peaks at the Davies point.

Compared with the Schwarzschild case, in which there is no such a turning critical point, in the charged case, clearly the difference is caused by the charge $Q$. So the presence of charge is the cause behind such a phase transition with nonthermal radiation. Since angular momentum behaves similarly to charge for black holes, we can anticipate this structure will persist when $J \neq 0$. 


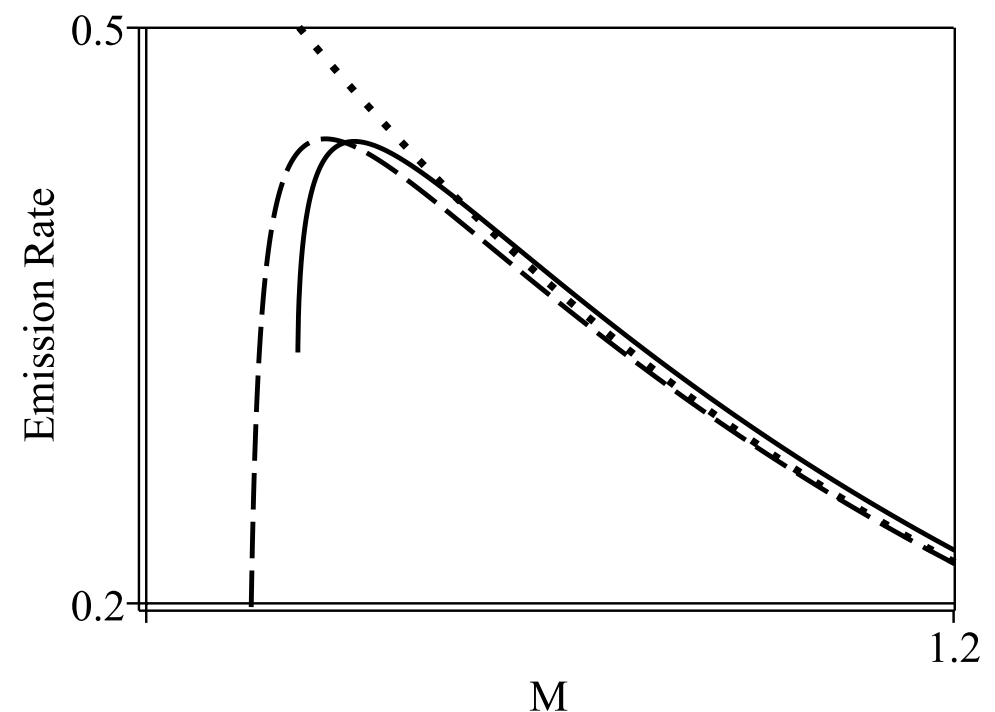

FIG. 5. Plots of (normalized) emission rate vs. mass for fixed $Q$. They are exaggerated to visualize the difference clearly. The solid line is for RN black hole with nonthermal contribution, while the dashed line is just for thermal part of RN black hole. The dotted line is the thermal part of Schwarzschild black hole for comparison. As $Q$ increases, the emission rate decreases and the peak location moves to the lower-right direction.

For the purpose of generalization, let's derive eq.(10) more formally. This can be done as follows: First, note that in terms of Wick rotation $t_{\mathrm{E}}=i t$

$$
\beta_{\mathrm{M}} \equiv 2 \int_{r_{\mathrm{in}}}^{r_{\mathrm{out}}} \frac{d r}{\left(\frac{d r}{d t}\right)}=2 \int_{r_{\mathrm{in}}}^{r_{\mathrm{out}}} \frac{d r}{\left(\frac{d r}{-i d t_{\mathrm{E}}}\right)}=-i \int_{0}^{\beta} d t_{\mathrm{E}}=-i \beta
$$

where $\beta$ is the inverse Hawking temperature, which can be shown by directly integrating along a geodesic that crosses the horizon. (See the Appendix A for the proof.) The factor 2 is present because one needs to cross the horizon back and forth to complete the period for the Euclidean time to be a temperature. Using this, we can identify

$$
\operatorname{Im} \int_{M}^{M-\omega} \frac{1}{2} \beta_{\mathrm{M}}(H) d H=\frac{1}{2} \int_{0}^{\omega} \beta\left(M-\omega^{\prime}\right) d \omega^{\prime} .
$$


Then, expanding in terms of $\omega^{\prime}$, from eqs.(2)(4) we can obtain

$$
\begin{aligned}
\beta_{\mathrm{eff}}(\omega) \omega & =\int_{0}^{\omega} \beta\left(M-\omega^{\prime}\right) d \omega^{\prime} \\
& =\int_{0}^{\omega} d \omega^{\prime}\left(\beta(M)+\left.w^{\prime} \frac{\partial \beta}{\partial \omega^{\prime}}\right|_{\omega^{\prime}=0}+\mathcal{O}\left(\omega^{\prime 2}\right)\right) \\
& =\omega \beta(M)+\left.\frac{1}{2} \omega^{2} \frac{\partial \beta}{\partial \omega}\right|_{\omega=0}+\mathcal{O}\left(\omega^{3}\right) \\
& =\omega \beta(M)+\frac{1}{2} \frac{\omega^{2}(\beta(M))^{2}}{M c_{Q}}+\mathcal{O}\left(\omega^{3}\right),
\end{aligned}
$$

where $\beta(M)=\beta_{H}$. Note that in the above derivation we have not specified what kind of black hole we use. So, we have a general theorem that for any black hole (including the case with $J \neq 0$, i.e. the $\mathrm{KN}$ black hole) which emits energy $\omega \ll M$, eq.(10) holds true.

The Helmholtz free energy cannot be used to check the stability of the system with respect to the radiation because it is constant for fixed Hawking temperature for which both $Q$ and $M$ need to be fixed. However, we can take an analogy of the idea of effective temperature and check the stability with respect to the radiation. For this purpose, let us define an effective Helmholtz free energy after the emission such that

$$
F_{\mathrm{eff}}:=M_{\mathrm{eff}}-T_{\mathrm{eff}} S_{\mathrm{eff}} .
$$

This can be used to compare the Helmholtz free energies before and after emission of radiation at fixed temperature. Then using the values after emission

$$
F_{\text {eff }}=M+\Delta M-\frac{1}{\beta_{\text {eff }}}(S+\Delta S)=F+\Delta F,
$$

where

$$
\begin{aligned}
\Delta M & \equiv-\omega<0, \\
\Delta S & \equiv-\beta_{\mathrm{eff}} \omega<0, \\
\Delta F & \equiv \frac{1}{2} \frac{S}{c_{Q}} \frac{\omega}{M}+\mathcal{O}\left(\omega^{2}\right)
\end{aligned}
$$

and $F$ is the usual Helmholtz free energy, hence $\Delta F$ measures the difference at the same Hawking temperature. In fact,

$$
F_{\text {eff }}=M-T_{\text {eff }} S .
$$

Note that the change of the Helmholtz free energy is given in terms of the specific heat in the leading order of $\omega / M$. In particular, $\Delta F=0$ at the critical point. $\Delta F<0$ for $c_{Q}<0$, which 
can be interpreted as that the black hole with nonthermal radiation is more stable so that black hole will keep emitting nonthermal radiation until it gets evaporated. However, for $c_{Q}>0, \Delta F>0$, which means the black hole before the nonthermal radiation is more stable, hence the nonthermal radiation will be suppressed, or there has to be another mechanism for more nonthermal emission. This will be addressed in section 5 again.

\section{DAVIES CRITICAL POINT FROM TUNNELING IN KERR-NEWMAN BLACK HOLE}

Next, we shall consider a more general case with $J \neq 0$. At the same time we shall allow charged particles[11][12] (also see [13] for an earlier attempt) as well as angular momenta to be emitted. The thermodynamic relation of a KN blackhole is given by

$$
d M=T_{H} d S+V_{H} d Q+\Omega_{H} d J
$$

where

$$
\begin{aligned}
T_{H}=\frac{1}{\beta_{H}} & =\frac{r_{+}-r_{-}}{A_{K}}, \\
S & =\frac{1}{4} A_{K}, \\
V_{H} & \equiv \frac{4 \pi r_{+} Q}{A_{K}}, \\
\Omega_{H} & \equiv \frac{4 \pi a}{A_{K}},
\end{aligned}
$$

where $a=J / M, A_{K}(M, J, Q)=4 \pi\left(r_{+}^{2}+a^{2}\right)$ and $r_{ \pm} \equiv M \pm \sqrt{M^{2}-Q^{2}-a^{2}}$.

Then, as shown in Appendix B, for emission of energy $\omega$, charge $q$, and angular momentum $j$, we have

$$
\begin{aligned}
-2 \operatorname{Im} I= & \Delta S \\
= & -\beta_{H}\left(\omega-q V_{H}-j \Omega_{H}\right) \\
& -\frac{1}{2} \frac{\omega^{2}}{M} \frac{\beta_{H}^{2}}{c_{Q}}-\frac{1}{2} q^{2}\left(\frac{\beta_{H}}{Q_{\kappa_{Q}}}-\frac{\beta_{H}^{2}}{\alpha_{Q} Q} V_{H}\right) \\
& -\frac{1}{2} j^{2}\left(\frac{\beta_{H}}{J \kappa_{J}}-\frac{\beta_{H}^{2}}{\alpha_{J} J} \Omega_{H}\right) \\
& -\omega \frac{q}{Q} \frac{\beta_{H}^{2}}{\alpha_{Q}}-q j\left(\frac{\beta_{H}^{2}}{S} \Omega_{H} V_{H}-\frac{\beta_{H}^{2}}{\alpha_{Q} Q} \Omega_{H}\right) \\
& -\omega \frac{j}{J} \frac{\beta_{H}^{2}}{\alpha_{J}}+\text { h.o. }
\end{aligned}
$$


where the following definitions of thermodynamic quantities for black holes are used:

$$
\begin{aligned}
\alpha_{Q} & \equiv \frac{1}{Q} \frac{\partial Q}{\partial T}: \text { charge expansion coefficient, } \\
\alpha_{J} & \equiv \frac{1}{J} \frac{\partial J}{\partial T}: \text { angular momentum expansion coefficient, } \\
\kappa_{Q} & \equiv \frac{1}{Q}\left(\frac{\partial Q}{\partial V_{H}}\right)_{T}: \text { charge "compressibility", } \\
\kappa_{J} & \equiv \frac{1}{J}\left(\frac{\partial J}{\partial \Omega_{H}}\right)_{T}: \text { angular momentum "compressibility". }
\end{aligned}
$$

Note that

$$
\begin{aligned}
& \frac{\beta_{H}}{Q \kappa_{Q}}-\frac{\beta_{H}^{2}}{\alpha_{Q} Q} V_{H}=2 \pi\left(1+\frac{1}{B^{1 / 2}}+\frac{Q^{2}}{M^{2}} \frac{1}{B^{3 / 2}}\right)>0, \\
& \frac{\beta_{H}}{J \kappa_{J}}-\frac{\beta_{H}^{2}}{\alpha_{J} J} \Omega_{H}=2 \pi \frac{M^{2}-Q^{2}}{M^{4} B^{3 / 2}}>0 \\
& \frac{\beta_{H}^{2}}{\alpha_{Q} Q}=-2 \pi \frac{Q\left(Q^{2}+a^{2}\right)}{M^{3} B^{3 / 2}}\left\{\begin{array}{l}
<0 \text { if } Q>0, \\
>0 \text { if } Q<0
\end{array}\right. \\
& \frac{\beta_{H}^{2}}{S} \Omega_{H} V_{H}-\frac{\beta_{H}^{2}}{\alpha_{Q} Q} \Omega_{H}=2 \pi \frac{Q a}{M^{3} B^{3 / 2}}\left\{\begin{array}{l}
>0 \text { if } Q>0, \\
<0 \text { if } Q<0,
\end{array}\right. \\
& \frac{\beta_{H}^{2}}{\alpha_{J} J}=-2 \pi \frac{a\left(2 M^{2}-Q^{2}\right)}{M^{4} B^{3 / 2}}<0,
\end{aligned}
$$

where $B \equiv 1-\left(Q^{2}+a^{2}\right) / M^{2}$ and $J>0$ is assumed without loss of generality. This shows that the only term which changes sign for nontrivial values of $Q$ and $J$ is the specific heat term. In this case the sign change occurs at

$$
\frac{Q^{2}}{M^{2}}=\frac{1}{4}\left(3-6 \frac{a^{2}}{M^{2}}-\frac{a^{4}}{M^{4}}\right),
$$

which identifies the Davies critical points[2]. As pointed out by Davies, this critical phenomenon takes place even for $Q=0$ at $a^{2} / M^{2}=J^{2} / M^{4}=2 \sqrt{3}-3$. In between, as $a^{2} / M^{2}$ varies from zero to $2 \sqrt{3}-3, Q^{2} / M^{2}$ varies from $3 / 4$ to zero.

One can also check the signs of other thermodynamic quantities and find

$$
\begin{aligned}
& \alpha_{Q}<0, \\
& \alpha_{J}<0 \\
& \kappa_{Q}>0 \text { if } Q>0,<0 \text { if } Q<0, \\
& \kappa_{J}>0 .
\end{aligned}
$$




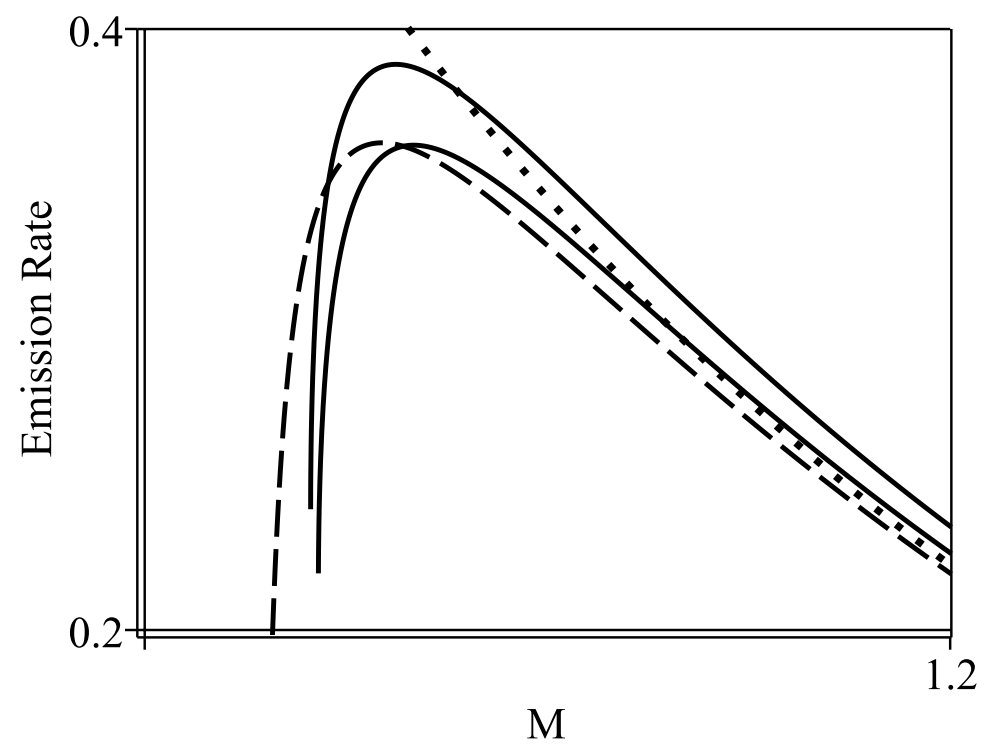

FIG. 6. Plots of (normalized) emission rate vs. mass for fixed $Q$ and $J$. Again, they are exaggerated to visualize the difference clearly. The lower solid line is for $\mathrm{KN}$ black hole with nonthermal contribution for $q=0=j$, and the upper solid line is for KN black hole with nonthermal contribution for $q \neq 0 \neq j$, $q Q>0$, while the dashed line is just for thermal part of $\mathrm{KN}$ black hole. The dotted line is the thermal part of Schwarzschild black hole for comparison. As $Q$ or $J$ increases, the emission rate decreases and the peak location moves to the lower-right direction.

In the extremal limit, they all approach to zero, including the specific heat, and, as $M \rightarrow \infty$, they all diverge. So, the existence of the Davies critical point is quite unique for the specific heat.

Even though $\omega, q, j$, are introduced independently, they cannot be arbitrary. This is because physically charges or angular momenta cannot be emitted without changing energy. For example, it is necessary that

$$
\left(\omega-q V_{H}-j \Omega_{H}\right)>0
$$

for the emission rate to make sense and that $\Delta S<0$. Otherwise, the emission rate diverges as temperature goes to zero. This provides us interesting bounds.

Let's consider $j=0$ case, first. Then

$$
\frac{\omega}{|q|}>\left|V_{H}\right|=\frac{|Q|}{M} \frac{1+\sqrt{B}}{(1+\sqrt{B})^{2}+a^{2} / M^{2}},
$$


for $q Q>0 .\left|V_{H}\right|<1$ and approaches zero as $M \rightarrow \infty$, hence this does not necessarily imply $\omega>q$. Nevertheless, this bound is quite significant, compared with $m_{e} / e \sim 10^{-21}$ or $m_{p} / e \sim 10^{-18}$. This means that charge has to be emitted with a sufficiently large energy. Not surprisingly, the larger $Q / M$ ratio is, the more energetically charges will be emitted. This also has a rather interesting alternative interpretation. Suppose $Q / M$ violates this bound for some charge emission, then the bad behavior of emission rate can be interpreted as black holes inability to sustain charge-to-mass ratio $Q / M$. There are two ways of lowering $Q / M$ : either emit charges or absorb more mass to increase $M$. What if not much matter is around the black hole to absorb? The only resolution will be to emit charges. In other words, this is a bound for a black hole how much charge it can sustain. Based on the classical argument, we know $Q / M<m / q$ is a limit for a black hole to absorb stationary charged particle. Here we have another bound beyond which a black hole cannot emit charges normally, but must get rid of them drastically. Note that, unlike the classical bound, the bound in the tunneling case is given in terms of emitting energy, not rest mass. If $q Q<0$, eq.(25) is always satisfied. This means that opposite charges can also be emitted and $Q$ can actually increase, although the emission rate is lower than that of $q Q>0$.

A similar bound can be obtained for the emission of the angular momenta. For $q=0$, since $j J>0$,

$$
\frac{\omega}{j}>\Omega_{H}=\frac{J}{M^{3}} \frac{1}{(1+\sqrt{B})^{2}+J^{2} / M^{4}} .
$$

To look into the next order contribution, we first need to impose eq.(25). Now the emission rate actually depends on the signs of $Q$ and $q$. The $q^{2}$ and $j^{2}$ terms always enhance the emission rate, but the contribution of $\omega^{2}$ term depends on the sign of specific heat and vanishes at the Davies point, where the emission rate peaks. If $q Q>0$, the last two terms with $q$ in the quadratic expansion enhance the emission rate. However, if $q Q<0$, they suppress the emission rate. The term with $j$ always enhances the emission rate since we assume $J>0$ and $j>0$.

In the $\mathrm{RN}$ case, we checked the stability of the black hole with respect to the radiation of energy, using the effective Helmholtz free energy. In the KN case, however, the effective Helmholtz free energy is not sufficient because of charge and angular momentum emissions. Note that the Gibbs free energy cannot be used to check the stability of a KN black hole with respect to the radiation either because it is constant for fixed $T_{H}, V_{H}$ and $\Omega_{H}$, for which 
all $M, Q, J$ are fixed.

So, we need to consider the effective Gibbs free energy analogously defined, like the effective Helmholtz free energy, in terms of effective quantities as

$$
G_{\mathrm{eff}}=M_{\mathrm{eff}}-T_{\mathrm{eff}} S_{\mathrm{eff}}-V_{\mathrm{eff}} Q_{\mathrm{eff}}-\Omega_{\mathrm{eff}} J_{\mathrm{eff}}
$$

then the difference between the effective Gibbs free energy and the usual Gibbs free energy at the same Hawking temperature reads, as derived in Appendix C,

$$
\Delta G \equiv G_{\mathrm{eff}}-G=\omega G_{\omega}+q G_{q}+j G_{j}
$$

where

$$
\begin{aligned}
G_{\omega} & \equiv-\frac{\pi^{2}}{\beta_{H}^{2}} \frac{A}{B^{3 / 2}}\left(2 M^{2}+Q^{2}+\frac{2 M^{2}}{B^{1 / 2}}\right), \\
G_{q} & \equiv \frac{\pi Q}{\beta_{H} B^{3 / 2}} \\
& \times\left(1+B^{3 / 2}-\frac{2 \pi}{\beta_{H}} \frac{Q^{2}+2 J^{2} / M^{2}}{M^{3}}\left(2 M^{2}+Q^{2}+\frac{2 M^{2}}{B^{1 / 2}}\right)\right), \\
G_{j} & \equiv \frac{\pi J}{\beta_{H} B^{3 / 2}}\left(\frac{1}{M^{2}}-\frac{2 \pi}{\beta_{H}} \frac{2 M^{2}-Q^{2}}{M^{5}}\left(2 M^{2}+Q^{2}+\frac{2 M^{2}}{B^{1 / 2}}\right)\right),
\end{aligned}
$$

where $B \equiv 1-\left(Q^{2}+J^{2} / M^{2}\right) / M^{2}$ as before and

$$
A \equiv 2 B^{3 / 2}+2-6 \frac{J^{2}}{M^{4}}-3 \frac{Q^{2}}{M^{2}}+\frac{J^{2} Q^{2}}{M^{6}} .
$$

Note that $A=0$ identifies the Davies critical points. One can show that $G_{j}<0$ for $J \neq 0$ always, however, the signs of $G_{\omega}$ and $G_{q}$ depend on $Q, J$ and $M$.

In summary, there are over all three different cases of $\Delta G$ depending on the variables, which are $M, Q, J$ and $\omega, q, j: \Delta G<0$ for all $M, \Delta G \gtrless 0$ for $M \lessgtr M_{0}$, where $M_{0}$ is another solution to $\Delta G=0$, or $\Delta G>0$ for some interval of $M$ while $\Delta G<0$ for the rest $M$. If $J=0, M_{0}=M_{c}$, but if $J \neq 0, M_{0}$ is different from $M_{c}$. The existence of opposite signs of $\Delta G$ indicates there are two different phases of KN black holes. The details are as follows.

If $J=0$, this describes the RN black hole with charge emission for $q \neq 0$ or without charge emission for $q=0$. In the $q=0$ case, $\Delta G$ correctly reduces to $\Delta F$ so that it simply reproduces the result of effective Helmholtz free energy case. So we shall consider $q \neq 0$ case here. For $J=0, G_{q}$ can be rewritten as

$$
G_{q}=\frac{\pi Q}{\beta_{H} B^{3 / 2}} \frac{2 M^{2}\left(1+B^{1 / 2}\right)-Q^{2}}{\left(2 M^{2}-Q^{2}+2 M^{2} B^{1 / 2}\right)\left(1+B^{1 / 2}\right)} A
$$


so that $G_{q}=0$ if $A=0 . G_{\omega}=0=G_{q}$, hence $\Delta G=0$ at $M=M_{c}$, that is, the Davies critical point. For $J=0$ and fixed $Q, G_{\omega} \gtrless 0$ for $M \lessgtr M_{c}$, while $G_{q} \lessgtr 0$ for for $M \lessgtr M_{c}$. So, if $\omega / q \geq 1, \Delta G \gtrless 0$ for $M \lessgtr M_{c}$. In the case $\omega / q<1$, there is a value $q^{\prime}$ such that, if $\omega / q<\omega / q^{\prime}<1, J=0, \Delta G>0$ for $M_{c}>M>M_{0}$, while, if $\omega / q^{\prime}<\omega / q<1, J=0, \Delta G>0$ for $M_{0}>M>M_{c}$, where $M_{0}$ is again another solution to $\Delta G=0$ and if $\omega / q=\omega / q^{\prime}<1$, $M_{0}=M_{c}$, hence $\Delta G \leq 0$. So, $M_{0}$ depends on the ratio $\omega / q$, which is another indication that $\omega$ and $q$ are not totally independent.

If $J \neq 0, G_{\omega}$ still vanishes at $M_{c}$, but $G_{q}$ vanishes at some other value $M^{\prime}$ different from $M_{c}$. For $j=0$ and $\omega / q>\omega / q^{\prime}, \Delta G \gtrless 0$ for $M \lessgtr M_{0}$ and $\Delta G=0$ for $M=M_{0}$. For $j=0$ and $\omega / q<\omega / q^{\prime}<1, \Delta G>0$ for $M_{0}>M>M_{\min }$, where $M_{\min }$ is the bound given by eq.(25) and $q^{\prime}$ in this case is given by $\Delta G=0$ for $M=M_{\min }=M_{\mathrm{ex}}$, where $M_{\mathrm{ex}}$ is the value of the extremal black hole such that $M_{\mathrm{ex}}^{2} \equiv \frac{1}{2}\left(Q^{2}+\sqrt{Q^{2}+4 J^{2}}\right)$.

If $J \neq 0$ and $j \neq 0$, if $\omega / q>\omega / q^{\prime}, \Delta G<0$ for all $M$, while if $\omega / q=\omega / q^{\prime}<1$ and $j<j^{\prime}$, $\Delta G>0$ for $M_{0}>M>M_{\min }$ and $\Delta G<0$ for $M>M_{0}$ or $M>M_{\min }$. However, as $j$ gets larger, the gap between $M_{0}$ and $M_{\min }$ shrinks, at that, if $j>j^{\prime}$ for some $j^{\prime}, \Delta G<0$ for all $M$ again.

If $J \neq 0$ and $q=0$, for $\omega / j>\omega / j^{\prime}, \Delta G \gtrless 0$ for $M \lessgtr M_{0}$, while for $\omega / j<\omega / j^{\prime}, \Delta G<0$ for all $M$, where $j^{\prime}$ is determined by $\Delta G=0$ for $M_{0}=M_{\text {ex }}$. The $Q=0$ and $J \neq 0$ case has a similar behavior, too.

\section{DISCUSSION}

We have shown the physical implication of the Davies critical phenomenon from the tunneling point of view. The sign of specific heat is related to the sign of the nonthermal contribution to the black hole radiation. We have also shown that the emission rate based on the tunneling peaks at (or near, if charge or angular momentum emissions are allowed) the Davies critical point. The argument based on the effective free energies show there are two phases, indicating whether the emission is spontaneous or not.

Negative specific heat is common in collapsing self-gravitating systems which are isolated[14], so the real question to address in the black hole context is what happens to the black hole when the specific heat is positive. A black hole can never be truly a stable thermodynamic system because either matter always falls in and/or radiations always come out. The 
equilibrium between falling matter and outward emission does not save the situation because of the case without matter available to fall and black hole solution does not distinguish it. If isolated, a black hole cannot evolve adiabatically without violating the charge conservation or angular momentum conservation. In case radiations come out at the same time matter falls, we cannot use tunneling argument to test the adiabatic evolution because the emission rate remains constant for any temperature, hence does not make sense. One could argue based on thermodynamic relations, using $0=d S=d M-V_{H} d Q-\Omega_{H} d J$, to check if there is a solution with a reasonable boundary condition. However, in the limit $Q$ and $J$ vanish, $M$ has to be constant. Since $M$ cannot be zero because, otherwise, it violates the unitarity, we end up with a Schwarzschild black hole whose specific heat is always negative. So the positive sign of the specific heat does not really imply its stability, unlike normal thermodynamic systems. Even in the extremal limit, it is not perfectly clear if the black hole is stable. (For example, see [15].) $T_{H}=0$, but we still have high charge-to-mass or angularmomentum-to-mass ratio so that it is not clear how it can sustain the zero temperature. We expect there should be no radiation, but the tunneling argument based on the WKB method fails in the extremal limit so that we cannot confirm, although it appears to be consistent. It will be interesting if one can check whether a black hole can really evolve into the extremal limit, or some kind of phase transition takes place at the extremal limit and it actually fails to be a black hole.

From the tunneling point of view, the positive specific heat indicates that the radiation is suppressed by the nonthermal contribution. The effective Helmholtz free energy leads to $\Delta F>0$, indicating this suppression does not take place spontaneously. We suspect that this signals there should be another phenomenon taking place presumably right outside of the horizon since it may not be an intrinsic black hole phenomenon, which will end up increasing the black hole radiation toward the thermal level direction, like that of Damour-Ruffini[8] and Blandford-Znajek[9]. Therefore, we also suspect that there might be a connection between Parikh-Wilczek's nonthermal radiation[6] and that of Damour-Ruffini[8] and BlandfordZnajek[9]. The effort to relate them is in progress and will be reported elsewhere.

\section{ACKNOWLEDGMENTS}

The author thanks L. Alvarez-Gaumé and S. Deser for helpful communications, and G. 
Siopsis for helpful discussions.

\section{Appendix A: Yet Another Derivation of Hawking Temperature}

The black hole temperature can be obtained by performing the Wick rotation of the metric so that the period of the Euclidean time along a circle can be identified as the inverse temperature. Consider a generic metric with the Euclidean signature

$$
d s^{2}=U(r) V(r) d t^{2}+\frac{1}{V(r)} d r^{2}+r^{2} d \Omega^{2}
$$

A godly traveler moving along a geodesic should be back to the original location after this time period, crossing the horizon back and forth. So we can compute the traveling period $\beta$ as

$$
\beta=\int_{0}^{\beta} d t=\oint \frac{d t}{d r} d r=2 \int_{P} \frac{d t}{d r} d r=2 \int_{P} \frac{d r}{\dot{r}},
$$

where $P$ denotes a geodesic path crossing the horizon and we have eq.(11) with $t \equiv t_{\mathrm{E}}$. Note that for $d s^{2}=0$ and $\theta=0=\phi$

$$
\frac{d t}{d r}=\sqrt{-\frac{g_{r r}}{g_{t t}}} .
$$

So we might be tempted just to use this, but in the Euclidean signature this is imaginary so that we need to be a little bit more cautious and justify it.

Without loss of generality and for convenience, we choose $\theta=0=\phi$, then nontrivial geodesic equations are

$$
\begin{aligned}
& 0=\ddot{t}+(\ln (U V))^{\prime} \dot{t} \dot{r}, \\
& 0=\ddot{r}-\frac{1}{2} V(U V)^{\prime} \dot{t}^{2}-\frac{1}{2} \frac{V^{\prime}}{V} \dot{r}^{2},
\end{aligned}
$$

where the dot denotes the derivative with respect to the affine parameter $\lambda$ and the prime denotes the derivative with respect to $r$. Eq.(A4a) can be integrated to

$$
U V \dot{t}=c_{1}
$$

where $c_{1}$ is an integration constant. Using this, eq.(A4b) can be written as

$$
0=\ddot{r}-\frac{1}{2} V \frac{(U V)^{\prime}}{(U V)^{2}} c_{1}^{2}-\frac{1}{2} \frac{V^{\prime}}{V} \dot{r}^{2} .
$$


Multiplying $2 \dot{r} / V$, we obtain

$$
0=\frac{d}{d \lambda}\left(\frac{\dot{r}^{2}}{V}\right)+c_{1}^{2} \frac{d}{d \lambda}\left(\frac{1}{U V}\right)
$$

hence

$$
\dot{r}^{2}+\frac{c_{1}^{2}}{U}=c_{2} V
$$

where $c_{2}$ is another integration constant. Using eq.(A5) and eq.(A8), we can perform the integration eq.(A2):

$$
\beta=2 \int_{P} \frac{d t}{d r} d r=2 \int_{P} d r \frac{c_{1}}{U V} \frac{1}{\sqrt{c_{2} V-c_{1}^{2} / U}}
$$

Note that we can choose $c_{2}=0$ for our geodesic path such that eq.(A3) is more or less justified. This integral is multi-valued, so we should choose the smallest nonvanishing value as the period. Then the integration simply becomes (assuming the pole is a simple pole, which is usually the case), using the Cauchy theorem after analytic continuation with a suitable path,

$$
\begin{aligned}
\beta & =\frac{2}{i} \int_{P} d r \frac{1}{\sqrt{U} V} \\
& =\frac{2}{i} 2 \pi i \operatorname{Res}_{r=r_{H}} \frac{1}{\sqrt{U} V} \\
& =4 \pi \lim _{r \rightarrow r_{H}}\left[\frac{d}{d r}(\sqrt{U} V)\right]^{-1} .
\end{aligned}
$$

The black hole temperature is, then, given by

$$
T_{H}=\frac{1}{\beta}=\frac{1}{4 \pi} \lim _{r \rightarrow r_{H}} \frac{d}{d r}(\sqrt{U} V)=\frac{1}{4 \pi} \lim _{r \rightarrow r_{H}} \frac{d}{d r} \sqrt{\frac{g_{t t}}{g_{r r}}} .
$$

For $U=1$, we have

$$
T_{H}=\frac{1}{\beta}=\frac{1}{4 \pi} \lim _{r \rightarrow r_{H}} \frac{d}{d r} g_{t t} .
$$

For KN black hole, set $\theta=0$, which in turn sets $g_{t \phi}=0$ (also set $g_{\phi \phi}=0$ at the end), then the metric satisfies the same geodesic equations eqs.(A4a,A4b). So we can still apply eq. (A11) to obtain the Hawking temperature for KN black hole as

$$
T_{H}=\frac{r_{+}-r_{-}}{4 \pi\left(r_{+}^{2}+a^{2}\right)}
$$




\section{Appendix B: Derivation of Eq.(20) for KN black hole}

In the WKB approximation, using the Hamilton-Jacobi theory, the emission of energy $\omega$, charge $q$, and angular momentum $j$, is given by[11][12]

$$
\operatorname{Im} I=\operatorname{Im} \int\left(H-p_{A} \dot{A}-p_{\phi} \dot{\phi}\right) d t
$$

where the generalized coordinates we use are $(r, A, \phi)$. Using eq.(18) and the fact that, as far as these generalized coordinates are concerned, $S, Q, J$ are corresponding independent variables, we can obtain the following identities these generalized coordinates should satisfy:

$$
\begin{aligned}
& \dot{r}=\frac{\partial H}{\partial p_{r}}=\frac{1}{\beta_{H}} \frac{\partial S}{\partial p_{r}} \\
& \dot{A}=\frac{\partial H}{\partial p_{A}}=V_{H} \frac{\partial Q}{\partial p_{A}} \\
& \dot{\phi}=\frac{\partial H}{\partial p_{\phi}}=\Omega_{H} \frac{\partial J}{\partial p_{\phi}} .
\end{aligned}
$$

Substituting the integrations $H=\int d M, p_{A}=\int d p_{A}$, and $p_{\phi}=\int d p_{\phi}$, we obtain

$$
\operatorname{Im} I=\operatorname{Im} \iint \frac{d r}{\dot{r}}\left(d M-V_{H} d Q-\Omega_{H} d J\right) .
$$

In fact, using eq.(B2a), this is actually equivalent to

$$
\begin{aligned}
\operatorname{Im} I & =\operatorname{Im} \int p_{r} \dot{r} d t \\
& =\operatorname{Im} \int p_{r} \frac{\partial H}{\partial p_{r}} d t \\
& =\operatorname{Im} \iint d p_{r} \frac{1}{\beta_{H}} \frac{\partial S}{\partial p_{r}} d t \\
& =\operatorname{Im} \iint \frac{d r}{\dot{r}} \frac{1}{\beta_{H}} d S .
\end{aligned}
$$

From eq.(B1) and eq.(B4), we can consistently identify $H$ as the Hamilton's characteristic function in this case.

Finally, using eq.(11), we can show that

$$
-2 \operatorname{Im} I=\Delta S \equiv \int d S=S(M-\omega, Q-q, J-j)-S(M, Q, J) .
$$

We can assume $J>0$ without loss of generality and that $j J>0$. However, the formula is valid for any signs of $Q$ and $q$, as long as $\Delta S<0$, hence we are not going to make any 
assumption on the signs of $Q$ and $q$ at this moment. Let's expand $\Delta S$ in terms of $\omega, q, j$, such that

$$
\begin{aligned}
\Delta S= & -\omega \frac{\partial S}{\partial M}-q \frac{\partial S}{\partial Q}-j \frac{\partial S}{\partial J} \\
& +\frac{1}{2} \omega^{2} \frac{\partial^{2} S}{\partial M^{2}}+\frac{1}{2} q^{2} \frac{\partial^{2} S}{\partial Q^{2}}+\frac{1}{2} j^{2} \frac{\partial^{2} S}{\partial J^{2}} \\
& +\omega q \frac{\partial^{2} S}{\partial M \partial Q}+q j \frac{\partial^{2} S}{\partial Q \partial J}+j \omega \frac{\partial^{2} S}{\partial J \partial M}+\text { h.o. }
\end{aligned}
$$

Now define the following thermodynamic quantities for black holes:

$$
\begin{aligned}
\alpha_{Q} & \equiv \frac{1}{Q} \frac{\partial Q}{\partial T}: \text { charge expansion coefficient, } \\
\alpha_{J} & \equiv \frac{1}{J} \frac{\partial J}{\partial T}: \text { angular momentum expansion coefficient, } \\
\kappa_{Q} & \equiv \frac{1}{Q}\left(\frac{\partial Q}{\partial V_{H}}\right)_{T}: \text { charge "compressibility", } \\
\kappa_{J} & \equiv \frac{1}{J}\left(\frac{\partial J}{\partial \Omega_{H}}\right)_{T}: \text { angular momentum "compressibility". }
\end{aligned}
$$

Then, from eq.(18), we can identify the following thermodynamic relations:

$$
\begin{aligned}
\frac{\partial S}{\partial M} & =\beta_{H}, \\
\frac{\partial S}{\partial Q} & =-\beta_{H} V_{H}, \\
\frac{\partial S}{\partial J} & =-\beta_{H} \Omega_{H}, \\
\frac{\partial^{2} S}{\partial M^{2}} & =-\frac{\beta_{H}^{2}}{M c_{Q}}, \\
\frac{\partial^{2} S}{\partial Q^{2}} & =-\frac{\partial}{\partial Q}\left(\beta_{H} V_{H}\right)=\frac{\beta_{H}^{2}}{\alpha_{Q} Q} V_{H}-\frac{\beta_{H}}{Q \kappa_{Q}}, \\
\frac{\partial^{2} S}{\partial J^{2}} & =-\frac{\partial}{\partial J}\left(\beta_{H} \Omega_{H}\right)=\frac{\beta_{H}^{2}}{\alpha_{J} J} \Omega_{H}-\frac{\beta_{H}}{J \kappa_{J}}, \\
\frac{\partial^{2} S}{\partial M \partial Q} & =\frac{\partial \beta_{H}}{\partial Q}=-\frac{\beta_{H}^{2}}{\alpha_{Q} Q}, \\
\frac{\partial^{2} S}{\partial Q \partial J} & =-\frac{\partial}{\partial Q}\left(\beta_{H} \Omega_{H}\right)=\frac{\beta_{H}^{2}}{\alpha_{Q} Q} \Omega_{H}-\frac{\beta_{H}^{2}}{S} \Omega_{H} V_{H}, \\
\frac{\partial^{2} S}{\partial J \partial M} & =\frac{\partial \beta_{H}}{\partial J}=-\frac{\beta_{H}^{2}}{\alpha_{J} J} .
\end{aligned}
$$


Thus we have

$$
\begin{aligned}
-2 \operatorname{Im} I= & \Delta S \\
= & -\beta_{H}\left(\omega-q V_{H}-j \Omega_{H}\right) \\
& -\frac{1}{2} \frac{\omega^{2}}{M} \frac{\beta_{H}^{2}}{c_{Q}}-\frac{1}{2} q^{2}\left(\frac{\beta_{H}}{Q \kappa_{Q}}-\frac{\beta_{H}^{2}}{\alpha_{Q} Q} V_{H}\right) \\
& -\frac{1}{2} j^{2}\left(\frac{\beta_{H}}{J \kappa_{J}}-\frac{\beta_{H}^{2}}{\alpha_{J} J} \Omega_{H}\right) \\
& -\omega \frac{q}{Q} \frac{\beta_{H}^{2}}{\alpha_{Q}}-q j\left(\frac{\beta_{H}^{2}}{S} \Omega_{H} V_{H}-\frac{\beta_{H}^{2}}{\alpha_{Q} Q} \Omega_{H}\right) \\
& -\omega \frac{j}{J} \frac{\beta_{H}^{2}}{\alpha_{J}}+\text { h.o., }
\end{aligned}
$$

which is eq.(20).

\section{Appendix C: Derivation of Eq.(29) for KN black hole}

The effective Gibbs free energy is defined by

$$
G_{\mathrm{eff}}=M_{\mathrm{eff}}-T_{\mathrm{eff}} S_{\mathrm{eff}}-V_{\mathrm{eff}} Q_{\mathrm{eff}}-\Omega_{\mathrm{eff}} J_{\mathrm{eff}}
$$

$M_{\text {eff }}$ and $S_{\text {eff }}$ can be identified as before in eq.(14), and the rest can be identified as following. The natural identification of $\Delta S$ in this case should be

$$
\Delta S=-\beta_{\mathrm{eff}}\left(\omega-q V_{\mathrm{eff}}-j \Omega_{\mathrm{eff}}\right)
$$

then from eq.(B6) we can read off the effective quantities as

$$
\begin{aligned}
\beta_{\mathrm{eff}} & =\frac{\partial S}{\partial M}-\frac{1}{2} \omega \frac{\partial^{2} S}{\partial M^{2}}-\left(q \frac{\partial^{2} S}{\partial M \partial Q}+j \frac{\partial^{2} S}{\partial J \partial M}\right), \\
\beta_{\mathrm{eff}} V_{\mathrm{eff}} & =-\frac{\partial S}{\partial Q}+\frac{1}{2} q \frac{\partial^{2} S}{\partial Q^{2}}+\frac{1}{2} j \frac{\partial^{2} S}{\partial Q \partial J} \\
\beta_{\mathrm{eff}} \Omega_{\mathrm{eff}} & =-\frac{\partial S}{\partial J}+\frac{1}{2} j \frac{\partial^{2} S}{\partial J^{2}}+\frac{1}{2} q \frac{\partial^{2} S}{\partial Q \partial J} .
\end{aligned}
$$

These identifications consistently truncate the effective Gibbs free energy to the effective Helmholtz free energy. And the definition of the effective Gibbs free energy eq.(C1) also actually reduces to

$$
G_{\mathrm{eff}}=M-T_{\mathrm{eff}} S-V_{\mathrm{eff}} Q-\Omega_{\mathrm{eff}} J
$$


Now we can compute the difference between the effective Gibbs free energy and the usual Gibbs free energy at the same Hawking temperature as

$$
\Delta G \equiv G_{\text {eff }}-G=\frac{S}{\beta_{H}^{2}} \Delta \beta-Q \Delta V-J \Delta \Omega,
$$

where

$$
\begin{aligned}
\Delta \beta= & \beta_{\mathrm{eff}}-\beta_{H} \\
= & \beta_{H}^{2}\left(\frac{1}{2} \frac{\omega}{M} \frac{1}{c_{Q}}+\frac{q}{Q} \frac{1}{\alpha_{Q}}+\frac{j}{J} \frac{1}{\alpha_{J}}\right), \\
\Delta V= & V_{\mathrm{eff}}-V_{H} \\
= & -\frac{1}{2} \frac{q}{Q}\left(\frac{1}{\kappa_{Q}}-\frac{\beta_{H}}{\alpha_{Q}} V_{H}\right)-\frac{1}{2} j \beta_{H} \Omega_{H}\left(\frac{V_{H}}{S}-\frac{1}{\alpha_{Q} Q}\right) \\
& -\frac{V_{H}}{\beta_{H}} \Delta \beta, \\
\Delta \Omega= & \Omega_{\mathrm{eff}}-\Omega_{H} \\
= & -\frac{1}{2} \frac{j}{J}\left(\frac{1}{\kappa_{J}}-\frac{\beta_{H}}{\alpha_{J}} \Omega_{H}\right)-\frac{1}{2} q \beta_{H} \Omega_{H}\left(\frac{V_{H}}{S}-\frac{1}{\alpha_{Q} Q}\right) \\
& -\frac{\Omega_{H}}{\beta_{H}} \Delta \beta .
\end{aligned}
$$

This can be rewritten as

$$
\Delta G=\omega G_{\omega}+q G_{q}+j G_{j}
$$

where

$$
\begin{aligned}
G_{\omega} & \equiv-\frac{\pi^{2}}{\beta_{H}^{2}} \frac{A}{B^{3 / 2}}\left(2 M^{2}+Q^{2}+\frac{2 M^{2}}{B^{1 / 2}}\right) \\
G_{q} & \equiv \frac{\pi Q}{\beta_{H} B^{3 / 2}}\left(1+B^{3 / 2}-\frac{2 \pi}{\beta_{H}} \frac{Q^{2}+2 J^{2} / M^{2}}{M^{3}}\left(2 M^{2}+Q^{2}+\frac{2 M^{2}}{B^{1 / 2}}\right)\right), \\
G_{j} & \equiv \frac{\pi J}{\beta_{H} B^{3 / 2}}\left(\frac{1}{M^{2}}-\frac{2 \pi}{\beta_{H}} \frac{2 M^{2}-Q^{2}}{M^{5}}\left(2 M^{2}+Q^{2}+\frac{2 M^{2}}{B^{1 / 2}}\right)\right),
\end{aligned}
$$

where

$$
\begin{aligned}
& B \equiv 1-\frac{Q^{2}+J^{2} / M^{2}}{M^{2}}, \\
& A \equiv 2 B^{3 / 2}+2-6 \frac{J^{2}}{M^{4}}-3 \frac{Q^{2}}{M^{2}}+\frac{J^{2} Q^{2}}{M^{6}} .
\end{aligned}
$$

[1] J. D. Bekenstein, Phys. Rev. D 7, 2333 (1973). 
[2] P. C. W. Davies, Rep. Prog. Phys. 411313 (1978); Proc. Roy. Soc. Lond. A 353, 499 (1977).

[3] P. Hut, Mon. Not. R. Astr. Soc. 180379 (1977).

[4] W. A. Hiscock and L. D. Weems, Phys. Rev. D 41, 1142 (1990).

[5] O. Kaburaki, I. Okamoto and J. Katz, Phys. Rev. D 47, 2234 (1993).

[6] M. K. Parikh and F. Wilczek, Phys. Rev. Lett. 85, 5042 (2000) [arXiv:hep-th/9907001].

[7] M. K. Parikh, arXiv:hep-th/0402166.

[8] T. Damour and R. Ruffini, Phys. Rev. Lett. 35, 463 (1975).

[9] R. D. Blandford and R. L. Znajek, Mon. Not. Roy. Astron. Soc. 179, 433 (1977).

[10] S. W. Hawking, Commun. Math. Phys. 43, 199 (1975) [Erratum-ibid. 46, 206 (1976)].

[11] J. Zhang and Z. Zhao, Phys. Lett. B 638, 110 (2006) [arXiv:gr-qc/0512153].

[12] R. Kerner and R. B. Mann, Phys. Lett. B 665, 277 (2008) [arXiv:0803.2246 [hep-th]].

[13] G. W. Gibbons, Commun. Math. Phys. 44, 245 (1975).

[14] D. Lynden-Bell, arXiv:cond-mat/9812172.

[15] O. Kaburaki, Gen. Rel. Grav. 23 (1996) 843. 